\title{
Antecedentes históricos en la descripción y del uso de Sophora toromiro (Phil.) Skottsb. (Fabaceae) en su hábitat natural: 1770-1948
}

\author{
Historical description and use of Sophora toromiro (Phil.) Skottsb. (Fabaceae) in the \\ wild: 1770-1948
}

\author{
JaIme ESPEJo ${ }^{1 *} \&$ Roberto Rodríguez ${ }^{2}$ \\ ${ }_{1}^{1}$ Programa de Postgrado. Facultad de Ciencias Forestales, Universidad de Concepción, Victoria 631, Casilla 160-C. Correo \\ 3. Concepción, Chile. \\ ${ }^{2}$ Departamento de Botánica. Universidad de Concepción, Victoria 631, Casilla 160-C, Concepción, Chile. \\ *jaimespejo@udec.cl
}

\begin{abstract}
RESUMEN
Sophora toromiro (Phil.) Skottsb. especie extinta en Isla de Pascua es descrita como un árbol de pequeñas dimensiones para mediados del siglo XVIII. Los bosquetes identificados por los relatos quedan ubicados mayoritariamente al interior de la isla, como en la cima de los cráteres de los volcanes de Rapa Nui. La madera fue ampliamente utilizada por los isleños principalmente en artefactos de índole sacra, sin embargo ya a fines del siglo XVIII la necesidad de madera y la pérdida de culto por las nuevas generaciones de Rapa Nui evidencian un cambio en su uso. Los últimos bosquetes como rebrotes fueron diezmados por herbivoría de masa ovejuna que fue desarrollada a gran escala en Isla de Pascua. En la actualidad esta especie está catalogada como extinta en su hábitat natural según la UICN.
\end{abstract}

Palabras clave: Sophora toromiro, conservación, Isla de Pascua.

\begin{abstract}
Sophora toromiro (Phil.) Skottsb. extinct species of Easter Island is described as a small tree to mid-eighteenth century. The clumps are identified by the first visitors and related mostly located within the island, as on top of the craters of the volcanoes of Rapa Nui. The wood was widely used by the islanders mainly in such sacred artifacts, however since the late eighteenth century the need for wood and the loss of worship for the new generations of Rapa Nui show a change in use. The last woods and shoots were decimated by herbivory that was developed sheepish mass scale on Easter Island. At present this species is listed as extinct in the wild by UICN.
\end{abstract}

KEYwords: Sophora toromiro, conservation, Easter Island.

\section{INTRODUCCIÓN}

La singularidad de la génesis de las islas como su aislamiento y en particular su flora y fauna hacen que la conservación de los recursos genéticos sean de interés tanto a la comunidad local, nacional y mundial (Hoffmann \& Marticorena 1987). Un ejemplo de lo anteriormente señalado es el caso de Isla de Pascua o Rapa Nui que está situada en el Océano Pacífico (2709'S; $\left.109^{\circ} 27^{\prime} \mathrm{O}\right)$, perteneciente al estado chileno y se encuentra separada de Chile continental por $3.525 \mathrm{~km}$.

La creación del Parque Nacional Rapa Nui queda registrada por el decreto número 103 del Ministerio de Tierras y Colonización (1935) por el estatus de conservación del único ejemplar de Sophora toromiro (Phil.) Skottsb., ubicado al interior del volcán Rano Kao.

El ocaso de la cultura Rapa Nui en el siglo XVIII, paralelamente a la deforestación de la Isla de Pascua, es un fenómeno que aún es motivo de investigación por grupos multidisciplinarios, Rolett \& Diamond (2004), Vargas et al. (2006), Hunt \& Lipo (2006), Rapaport (2006), Hunt (2007). Sin duda el aumento demográfico, el cambio en el uso del suelo y quemas contribuyeron a la pérdida de los ecosistemas de la isla. Esto es corroborado por registros de polen Flenley \& King (1984), Dransfield et al. (1984), Flenley et al. (1991) y los que informan de una rica flora existente antes del arribo de la cultura polinésica a Rapa 
Nui circa 800-1200 aD. Orliac \& Orliac (1998), Vargas et al. (2006), Hunt \& Lipo (2006) y Flenley (2007).

Una de las especies que persistió en pequeñas poblaciones en la isla hasta mediados del siglo XIX fue Sophora toromiro, la que revestía singular importancia para la población Rapa Nui según relatos de los primeros cronistas que visitaron la isla. La escasa literatura existente sugiere que esta especie pudo persistir en su hábitat en la forma de rebrotes en algunos sectores de la isla hasta finales del siglo XIX, y que éstos fueron destruidos en los periodos de explotación y colonización (1872-1888) y finalmente por el mal manejo administrativo a que Rapa Nui fue sometida por sus arrendatarios, Merlet y Cía. (1895-1953), conocidos como Compañía Explotadora de Isla de Pascua. La especie está extinta hace más de cincuenta años luego de la tala del último ejemplar,que crecía en una ladera interior del volcán Rano Kao. Las semillas colectadas a fines de los años 50 han dado origen a plantas que están resguardadas en jardines botánicos de Europa, Australia y Chile (Mauder et al. 1999, 2000).

En el ámbito de la conservación se han realizado innumerables esfuerzos por introducir esta especie en su hábitat que datan desde 1965 y todos han fracasado. El último programa para reintroducir la especie en Isla de Pascua fue realizado por la Agrupación para el Manejo del Toromiro (TMG) en 1998.

El presente documento tiene como objetivo compilar la documentación existente sobre $S$. toromiro entre los años 1770 y 1950 con un enfoque en la descripción de su hábitat y el uso de la especie en las fechas indicadas.

\section{Primeros Relatos}

La observación realizada por Francisco Antonio de Agüera y Infanzón en 1770 durante la visita de la fragata Santa Rosalía, aparece como la primera descripción de Sophora toromiro: "pero hay un montón de arbustos como un cepillo de madera de una especie de poco más de una estada en la altura, que ofrece poca obstrucción al paso, ya que no son densas ni tienen espinas. Estos árboles son muy similares, a las mimosas y tamarindos".

En 1774 se realiza una descripción más detallada por los botánicos Johann Reinhold Forster y Johann Georg Adam Forster que viajaban comisionados en el "HMS Resolution" comandado por James Cook: "Durante toda esta excursión, asi como durante la que hicieron el día precedente, solamente vieron dos o tres arbustos. La hoja y semilla de ellos (llamada por los isleños torromedo) eran bastante parecidas a las de la arveja común; pero la vaina se parecía más, en tamaño y forma, a la del tamarindo. La semilla tiene un gusto amargo y desagradable, y cuando vieron los indigenas que nuestras gentes las masticaban, hicieron señas para que la escupieran; dedúcese de aqui que eran consideradas por ellos como venenosas. La madera es de un color rojizo y bastante dura y pesada; pero muy sinuosa, delgada y corta, no excediendo el tronco de seis o siete pies de altura [sic] (Cook 1821).

J.Reinhold Forster, entrega un documento sobre el vocabulario de Isla de Pascua en el que figura la mimosa como Mimosa silignis articulates tetragloboides, Foerster (2012). La muestra colectada se encuentra depositada en el Museo Británico de Historia Natural (NH).

II. UN SIGLO MÁS TARDE

El padre (SSCC) H.Roussel (1869) en sus notas descriptivas de Rapa Nui escribe "Sólo la costa estaba habitada y los grupos de mahute, toromiro y hau que están esparcidos sugieren una población de no más de cinco mil personas con un promedio de cinco o seis personas por cabaña". Palmer (1870a) oficial y médico cirujano del "HMS Topaze" hace un comentario sobre la vegetación de Rapa Nui,"Habia troncos provenientes de grandes árboles, Edwardsia, palma de coco e hibiscos en descomposición en los mismos lugares, cuando visitó la isla, La Perousse dejó árboles frutales con los habitantes y no hemos visto ningún indicio de estos. Por el tamaño de algunas palas y rapas, los árboles grandes han existido. Ahora mismo la única aproximación para bosque se encuentra en los rincones protegidos, como arbustos de 10 a 12 pies de alto, de Hibiscus, Edwardsia, Broussonetia, etc... La tasa de crecimiento de estas es extremadamente lenta". El mismo Palmer (1870b) prosigue su relato en lo que se refiere a las pequeñas esculturas (Kava Kava).“Las de data más moderna son los de madera de ídolos del hogar....... Estos son muy diversos, muchos peculiarmente grotescos, algunos que vi son de edad inmemorial y algunos hechos sólo hace algunos unos meses. Estos están tallados en forma humana, en general son indudablemente de sexo masculino.....Su dimensión es de un pie o más de largo, hecho de una madera oscura y compacta (toromiro, una Edwardsia); el perfil fuertemente aguileño, los labios separados, a fin de dar una sonrisa; globos oculares de obsidiana; un mechón en la barbilla; las orejas con lóbulos largos y dilatados, la figura un poco aovada con los brazos a los lados y las manos planas en el lado de los muslos".

Gana (1870), comisionado por el Gobierno de Chile y responsable de anexar Isla de Pascua, comenta: "También se ven de trecho en trecho unos pequeños enramados de un arbolito llamado toromiro. Este arbolito, que debe elevarse tanto como la acacia australiana, a una de cuyas familias pertenece, ha suministrado troncos de 50 centímetros de diámetro. Todas las figuras e instrumentos de madera que hai en la isla de fecha inmemorial, son de este árbol. Al presente no hai un solo tronco en todo el país. Los retoños del toromiro proporcionan a los indios las barillas para sus rucas i las hastas para sus lanzas de guerra. Los lóbulos o vetas de esta madera tienen mucha semejanza con los del cedro, $i$ juzgando que pudiera crecer en nuestros campos $i$ de ser útil para los ebanistas llevo un poco de semilla para su propagación [sic]". 
Philippi (1873) señala: "No hai ningún árbol en la isla, $i$ solo tres arbustos. El primero es el toromido, que Forster, escribe torromedo con la ortografía inglesa, i que debe pronunciarse toromido, llama una Mimosa, en lo que lo ha seguido La Perouse; pero que es evidente una Edwardsia, como lo prueban los frutos traídos al museo por el Sr. Gana, pudiendo llamarse Edwardsia toromido. Según Cook, llegando solo a la altura de ocho a nueve pies, i solo algunos de los troncos inclinados hacia la raíz tenían el grueso del muslo. El señor Gana dice que ha suministrado troncos de 50 centímetros de diámetro, pero que al presente no hai un solo tronco en toda la isla. Todas las figuras e instrumentos de madera, las masuas o clavas, los patouspatous (otra clase de clava), las planchas para sus piraguas, son de esta madera. Los retoños proporcionan a los isleños las varillas para sus rucas o chozas, $i$ las astas para sus lanzas de guerra. Esta madera es de un color rojizo, de un grano fino e igual, bastante dura i pesada, sumamente diferente de la madera de nuestro pelú (Edwardsia macnabiana), pero mui torcida i suministra solo pequeños pedazos [sic]".

Pinart (1877) entrega la siguiente información: "Muchos de los isleños llevan pequeñas estatuillas, generalmente tallada en un tipo de Mimosa que es bastante común en la isla. Ellos valoran estas estatuillas altamente, como lo verificamos cuando intentamos, con dificultades, para conseguirla por tabaco. Las figuras representan tanto a hombres y mujeres, los Kanacas las guardan en bolsitas de lino o algodón. Algunas de las estatuillas no tienen cuello si una enorme cabeza con cabellera cuidadosamente representada". El mismo autor, entrega dos indicaciones sobre la distribución de poblaciones de Sophora toromiro (Phil.) Skottsb., la primera para el sector del Rano Raraku: "En los lados del cráter, había numerosas plantas de dracena, helechos, un tipo de acacia de flores amarillas, un grupo grande de algarrobos y un considerable número de viñas lagenaria"; o la segunda para el interior del volcán Rano Kau: "En frente lejos, al interior de la isla parece más vasta y oscura, con un conjunto árboles de mimosa y moreras que se inclinan por la tormenta."

Toro (1893) relata en su estadía: "La vegetación natural de la isla es bien pobre, no pasando de los tres clases de arbustos de que habla el doctor Philippi. De estos es el principal el toromiro (no torromido) que en otro tiempo formó sin duda bosques, pues en diversas partes de la isla se ven todavía innumerables $i$ tupido troncos secos de dos a tres metros de altura.

...Parece indudable que estos bosques remanentes han desaparecido principalmente de la introducción de animales vacunos $i$ ovejunos, que han quebrado las plantas o las han comido la corteza.

...Aquellos troncos secos proveen a los pobladores de la leña necesaria para sus menesteres domésticos, no sin trabajo a causa de las distancias i de la falta de vehículos. Un carretón adquirido del Sr. Salmon por un canaca a prestado buenos servicios en el acarreo de leña".

\section{ReLATOS MÁs RECIENTES}

Fuentes (1913a) entrega su visión de la flora de Rapa Nui: "Los indígenas no han cultivado árboles madereros, a pesar de su importancia para las construcciones. El toromiro esta por extinguirse alli a causa de los animales herbivoros $i$ por la explotación desmedida que se ha hecho de dicho arbolillo para construir herramientas, remos, estatuillas, etc...". Además entrega la hipótesis del origen de esta especie: "Una tradición dice que los primitivos canacas introdujeron la caña de azúcar, Mahute, Toromiro, etc.... Pero es más probable que las corrientes del océano hayan llevado los frutos flotadores del Toromiro desde el sur de Chile hasta Juan Fernández, Pascua, Nueva Zelanda o vice y versa" (Fuentes 1913b).

Skottsberg en su visita a la Isla en 1917 comenta: "Los árboles enanos ya existentes que ya se ha comentado quedan confinados al cráter del Rano Kao y están al borde de la extinción. Ellos crecen no lejos del lago, donde la pendiente está cubierta por grandes bloques que impiden a las ovejas llegar a ellos. El Sr. Edmund me habló de algunos de estos árboles que crecen a lo largo del empinado acantilado de la punta Este, por desgracia no fue posible visitar el lugar, espero que en el futuro algún naturalista lo hará" [sic] (1953).

Oyarzún (1921) afirma que todos los objetos de madera que confeccionan los naturales de Pascua, particularmente los pequeños mohais, los llaman toromiro.

Macmillan (1924) relata: "Mi guía recordaba haber visto cuando era un muchacho, árboles de sándalo y toromiro, árboles de $45 \mathrm{~cm}$. de diámetro en Anakena".

Metráux (1950) integrante de la expedición FrancoBelga de 1934, señala en su estudio etnográfico realizado en Isla de Pascua: "Los espacios deshabitados se hallan llenos de arbustos enclenques, cuyas ramas bajas y retorcidos no parecian de gran utilidad para los hombres. Roggeveen, González, Cook y La Perouse se intrigaron por esta falta de árboles de gran tamaño, que según ellos, impedía el desarrollo de una civilización de que la admiran, por otro lado, toda la salvaje grandeza. Cuando los primeros polinesios abordaron la isla hallaron en ella, sin duda, una especie de parque natural, lleno de boscajes de toro-miro (Sophora toromiro).

Este arbusto raquítico les proporcionó durante siglos la madera en la que tallaron la mayor parte de sus famosas imágenes ancestrales, y sus objetos ceremoniales. El toromiro ha seguido el destino de aquellos hombres que lo adoptaron para expresar su sentido de belleza. Ha desaparecido de la isla durante la mitad del siglo pasado, bajo el diente de cabras y los carneros, con excepción de un ejemplar único que ha sobrevivido en el interior del volcán Rano-kao, en un sitio de difícil acceso. En la época de nuestra visita a la isla, los indígenas vigilaban su 
crecimiento con mirada ambiciosa, esperando el momento propicio para echarla abajo y expenderlo convertido en estatuillas y otros "curios" [sic].

Lavachery (1935) realiza una descripción sui generis del último toromiro en el volcán Rano Kao: "Uno de los toromiros se esconde al final de una bananera densa que crece entre las piedras cubiertas de líquenes. Uno se resbala, camina a cuatro patas. En fin: el toromiro tiene la altura de un hombre y las delicadas hojas, sus racimos de flores amarillas, sus vainas colgantes, llenas de granos blancos y redondos, recordándonos la acacia que es uno de sus parientes. Su tronco, gordo como el brazo y chueco. Da la impresión de tener una fuerza ruda, se diría que musculosa. Nosotros lo fotografiamos e íbamos a cortarle una rama y colectar sus semillas. Será que veremos algún día florecer un toromiro en una sierra europea?"

El padre capuchino Sebastián Englert (1948) en sus investigaciones sobre la historia y antropología de Rapa Nui, entrega la distribución de Sophora toromiro: "Antes crecía en muchas partes, especialmente en la costa norte; no habia verdaderos bosques, pero si, muchos pequeños grupos de toromiro. Hoy queda un solo ejemplar en el cráter del volcán Rano Kau". El padre Englert entrega más antecedentes de la utilización de la madera de S.toromiro: "Los medios de labranza eran sencillísimos. No habia animales de trabajo, ni arado ni otros instrumentos de fierro como el azadón o rastrillo, sino únicamente dos clases de palos puntiagudos de toromiro: akaúve y oka.

“...El akaúve era un palo grueso y largo que se empleaba para abrir hoyos grandes y profundos en la plantación de camotes y de caña de azúcar. Oka era un palo delgado y menos largo que servía para limpiar la tierra de pastos, aporcar y plantar taro, plátanos, ñames y otras plantas que no necesitan hoyos profundos. Para dar más dureza y resistencia a las puntas del akaúve y oka las chamuscaban con fuego".

"...Las pequeñas embarcaciones que estaban siempre en uso, en el tiempo de Roggeweeny de otros europeos y hasta el final de la era antigua eran los vakaana que significa embarcación con balancín. El bote vakaana, se hacía de una canoa, tronco de toromiro u otro árbol y de pedazos de tabla que formaban el costado. La canoa se llamaba hakari o te vaka y su costado kaokao". Las tablas inscritas o kohau motu morongorongo, textos de la antigua civilización Rapa Nui, son mencionadas por el padre Englert: “...Solo después de adquirir algún perfeccionamiento, los alumnos escribian sobre las tablillas de madera con preferencia de madera de toromiro, empleando para estos grabados puntiagudas obsidianas o afilados dientes de tiburón".

\section{COMENTARIOS}

Según los relatos anteriormente expuestos, la visión o percepción de los primeros visitantes a Rapa Nui es la ausencia de árboles. Sin embargo, la vegetación más reconocida y reportada es la arbustiva en que $S$. toromiro es la más notable por los múltiples usos por parte de la etnia Rapa Nui (Gusinde 1920, Englert 1948).

Es muy probable que los individuos de S. toromiro de mayor envergadura hayan sido talados y que las dimensiones máximas hayan sido de no más de dos metros de altura, con perímetro entre los 15 a $30 \mathrm{~cm}$, según las primeras observaciones realizadas por los botánicos del HMS Resolution. Aquí aparece un error la medida del diámetro del fuste de $S$. toromiro entregada por Gana (1870) y Macmillan (1924), la lectura correcta debe estar referida al perímetro o bien el diámetro señalado pueda ser inferencia a partir de la observación de algunos ejemplares excepcionales. El hábito de crecimiento (fenotipo) de esta especie puede estar influenciado por las corrientes de viento y hayan configurado la denominación de "achaparrado" como lo menciona Pinart (1877) y Chauvet (1935).

Las recopilaciones por vía oral de los últimos Rapa Nui rescatadas por Englert (1948) apuntan a que en el área norte de la isla se encontraban las poblaciones de S.toromiro y concuerdan con las muestras de polen y fragmentos de madera de esta especie reportadas por Flenley \& King (1984) y Orliac \& Orliac (1998).

Es lógico pensar que las pequeñas poblaciones de esta especie ubicadas en las proximidades de los asentamientos Rapa Nui fueron las más afectadas y que las poblaciones remanentes quedaron confinadas a sectores de difícil acceso como las laderas interiores con pendiente como es el caso del volcán Rano Kao.

Sophora toromiro, era parte del sotobosque de los palmares de Niu (Paschalococos disperta) propuestos por Flenley \& King (1984), Flenley et al. (1991) Rull et al. (2010), Horrocks et al. (2013). De acuerdo con lo señalado anteriormente, el hábitat original de esta especie para la data revisada pueda ser catalogado como de bosquetes en el cual su ubicación en el estrato entra en la categoría codominante y tolerante. Sin embargo, los mismos autores sugieren que la distribución de $S$. toromiro haya incluido sectores mésicos desprovistos de palmeras.

Los registros o escritos entregados por los primeros navegantes occidentales aportan información importante sobre el uso y la distribución de esta especie. La madera de S. toromiro, según los antecedentes recopilados, suministró una gran gama de artefactos y utensilios propios de la cultura que colonizó la isla como lo reitera Gana (1870). Es indudable que los recursos vegetales de la isla no pudieron dar sustento a una población muy grande cuyo crecimiento tuvo un efecto gravitante en la demanda de recursos por parte de los isleños. Es altamente probable, además, que la demanda energética haya sido suministrada mayoritariamente por Sophora toromiro y Thespesia populnea (L.) Sol. ex Correa (Mako'i); al parecer la alta densidad de la madera de $S$. toromiro es un buen indicador de lo anteriormente señalado. 
Es interesante recalcar que en el rescate de las notas de Gana (1870) y Lavachery (1935) aparecen registros de colecta de semillas de $S$. toromiro las que requieren un estudio de seguimiento, especialmente la colecta de Gana ya que la mayoría de las ejemplares que crecen ex situ provienen de colecciones realizadas a fines de 1950 y han sido cosechadas del último ejemplar.

\section{DISCUSIÓN}

Los escasos registros escritos referentes a S.toromiro por parte de naturalistas y visitantes de Rapa Nui a mediados del siglo XVIII hasta el siglo XIX tienen relevancia tanto para el conocimiento del hábitat natural como para la utilización de esta especie por parte de los antiguos Rapa Nui.

Los cambios en la composición del paisaje son propuestos a partir de estudios paleobotánicos (Flenley \& King 1984). Estos evidencian que el uso de fuego fue una práctica recurrente en las islas de la Polinesia (Nunn 1990, Kirch \& Ellison 1994), y Rapa Nui no fue la excepción. La habilitación de sitios para la agricultura está directamente relacionada con el crecimiento demográfico y es el origen de estas perturbaciones en la fisonomía del paisaje de Rapa Nui. Prospecciones realizadas en el sector de Maunga Orito sugieren una secuencia de labranzas agrícolas para la subsistencia de la población local (Stevenson et al. 2006).

Levantamientos arqueológicos sistemáticos realizados en la isla señalan que las trazas de $S$. toromiro son una constante en los fogones conocidos como umu o umu pae (C. Cristino, com. pers.). Es un hecho que Sophora toromiro fue parte de la matriz energética por parte de sus habitantes puesto que el crecimiento demográfico sostenido, que llegó hasta 90 habitantes por $\mathrm{km}^{2}$, la sobreexplotación de los recursos naturales (Vargas et al. 2006) y los cambios climáticos con altas temperaturas y a continuación la aridez de los suelos (Nunn \& Britton 2001) como el drenaje excesivo de éstos (Cauwe 2012), fueron factores importantes que contribuyeron a la pérdida de la flora de la isla.

Otro factor que incidió en la desaparición de los últimos ejemplares de esta especie fue el ramoneo por parte de animales, esto es descrito por Toro, quien detalla en su informe que en la isla para 1892 se contaba con una masa de animales en torno a las 18.000 cabezas, principalmente ovejas y Metráux señala que para 1934 el número de animales ya estaba en el orden de las 40.000 .

No existen evidencias de la existencia de árboles de gran tamaño de Sophora toromiro para el periodo recopilado (1770-1948). El fenotipo queda definido para este periodo y a la fecha como el de un arbusto o árbol de pequeñas dimensiones, lo que está corroborado por ejemplares cultivados en jardines botánicos que tienen más de 50 años. Lo anteriormente señalado plantea inquietud con las recopilaciones orales de los Rapa Nui ("existencia de bosques de gran tamaño"). La escases de madera y su importancia lo refleja Metráux (1950): "La Isla de Pascua es tal vez una de las pocas regiones del mundo donde los sueños de fortuna se concretan en piezas de madera" [sic].

$\mathrm{Si}$ bien son escasos los artefactos originales confeccionados con madera de $S$. toromiro en Isla de Pascua, Orliac (2007) encontró que un 56\% de 200 piezas conservadas en museos y colecciones particulares estaban fabricadas con madera de esta especie. Los relatos de Palmer (1870) y Pinart (1877) señalan que las estatuillas llamadas Moai tangata y Moai kava kava eran portadas por los isleños y estaban presentes en la mayoría de las viviendas, lo anteriormente señalado es confirmado por Orliac (2007), quien recalca que los artefactos de mayor importancia o de culto estaban confeccionados con madera de Sophora toromiro. La importancia de éstos quizás ya se perdió en la memoria colectiva de la etnia Rapa Nui.

\section{CONCLUSIONES}

Queda en evidencia la importancia de $S$. toromiro en la cultura Rapa Nui, lo que refuerza la idea de que esta especie debe estar presente en la actual fisionomía de Isla de Pascua. Todos los avances en la investigación tanto del ámbito de conservación genética, la ecología del paisaje y de nuevas técnicas de establecimiento deben asegurar la conservación de esta especie tan singular como es el caso de Sophora toromiro.

\section{AGRADECIMIENTOS}

A Catherine Orliac, del Centre Nacional de la Recherche Scientifique (Francia), por aportar sus documentos como bibliografía, a Claudio Cristino del Centro de Estudios de Isla de Pascua de la Universidad de Chile, por la revisión del documento y entrega de antecedentes no publicados a la fecha, a Cristián Echeverría del Laboratorio de Ecología del Paisaje (LEP) de la Facultad de Ciencias Forestales de la Universidad de Concepción, por sus comentarios y sugerencias, como a dos correctores anónimos en la revisión del presente documento. A Forestal Mininco S.A por el apoyo otorgado a J. Espejo en el desarrollo de su programa de Doctorado.

\section{BIBLIOGRAFÍA}

AgÜERA, F. \& A. Infanzón. 1770. Journal of the principal occurrences during the voyage of the frigate Santa Rosalia from El Callao de Lima to the island of David and thence to San Carlos de Chiloe. Eastern Island: The First Three Expeditions 1722-1774. (Editor E. Ruiz Tagle), Rapanui 
Press. 65 pp.

Caume, N. 2012. Isla de Pascua (Diez años de excavaciones reconstruyen su historia). El Gran Tabú. Editions Versant Sud- Lovaina. 157 pp.

Chauvet, S. 1935. La isla de Pascua y sus misterios. Reedición Rapanui Press. 411 pp.

Cook, J. 1821. The three voyages of Captain Cook round the World. Printed for Longman, Hurst, Reese, Orme and Brown.London. Vol. III, Book II, Chap. VIII. 286 pp.

Dransfield, J., J.R. Flenley, S.S. King, D.D. Harkness \& S. Rapu. 1984. A recently extinct palm from Eastern Island. Nature 312: 750-752.

Englert, S. 1948. La Tierra de Hotu Matu'a: historia, etnología y lengua de Isla de Pascua. Editorial San francisco. Padre Las Casas. Villarrica Chile. 533 pp.

FLENLEY, J.R. 2007. A Palinologist Looks at the Colonization of the Pacific. In proceedings: Migration, Identity, and Cultural Heritage (The Gotland Papers). Gotland University, Sweden. 15-34.

FLENLEY, J.R. \& A.S.M. KING. 1984. Late Quaternary pollen records from Eastern Island. Nature 307: 47-50.

Flenley, J.R., S.King, J.T, Jakson, J.T. Teller \& M.E. Prentice. 1991. The Late Quaternary vegetational and climatic history of Eastern Island. Journal of Quaternary Science 6(2): 85-115.

Foester, R. 2012. Rapa Nui, primeras expediciones europeas. En Anexo: Diccionario de Johann Reinhold Forster. Editorial Rapanui Press. Santiago, Chile. 270-272.

Fuentes, F. 1913a. Reseña botánica sobre la isla de Pascua. Boletín Museo Nacional de Historia Natural 5(2): 320-327.

Fuentes, F. 1913b. Reseña botánica sobre la isla de Pascua. Instituto Central Meteorológico y Geofísico de Chile 4: $1-9$.

GanA, I.L. 1870. Descripción científica de la isla de Pascua. Memoria de la Marina 1870: 90-109.

Gusinde, M. 1920. Bibliografía de la isla de Pascua. Publicaciones del Museo de Etnología y Antropología de Chile, Tomo 2(2): 201-260.

Hoffmann, A. \& C. Marticorena. 1987. La vegetación de las Islas Oceánicas Chilenas. En: J.C. Castilla (ed.), Islas Oceánicas Chilenas: Conocimiento Científico y Necesidades de Investigaciones. Ediciones Universidad Católica de Chile. 127-165.

Horrocks, M., M. Marra, W.T. Baisden, J. Flenley Feek, L. González Nualart, S. Haoa Cardinali \& T. Edmunds Gorman. 2013. Pollen, phytoliths, arthropods and high resolution $14 \mathrm{C}$ sampling from Rano Kau, Eastern Island: evidence for late Quaternary environments, ant (Formicidae) distributions and human activity. Journal Paleolimnology. DOI 10.1007/S10933-013-9735-5.

Hunt, T. 2007. Rethinking Eastern Island ecological catastrophe. Journal Archeological Science 34: 485-502.

Hunt, T. \& C. LiPO. 2006. Late Colonization in Eastern Island. Science 311: 1603-1606.

KirCh, P. \& J. ElLison. 1994. Paleoenvironmental evidence for human colonization of remote Oceanic islands. Antiquity 68: 310-321.

Lavachery, H. 1935. Ile de Paques. Edition Bernard Grasset. Paris, 299 pp.

MacMillan, J. 1924, The Riddle of the Pacific. Reeditado por
Eduardo Ruiz-Tagle Eyzaguirre. Rapanui Press. 312 pp.

Mauder, M., A. Culham, A. Bordeau, J. Allainguillaume \& M. Wilkinson. 1999. Genetic diversity and pedigree for Sophora toromiro (Leguminosae): a tree extint in the wild. Molecular Ecology 8: 725-738.

Mauder, M., A. Culham, B. Alden, G. Zizka, C. Orliac, W. Lobin, A. Bordeau, J.M. Ramírez \& S. Glissmann-Gough. 2000. Conservation of the Toromiro Tree: Case Study in the Management of the Plant Extint in the Wild. Conservation Biology 14(5): $1341-1350$.

Metráux, A. 1950. Isla de Pascua. Editado por el Fondo de Cultura Económica. México. 264 pp.

NunN, P. 1990. Recent Environmental Changes on Pacific Islands. The Geographical Journal 156(2): 125-140.

NunN, P. \& J. Britton. 2001. Human Environmental Relationiships in the Pacific Islands around a.D 1300. Environmental and History 7: 3-22.

Orliac, C. 2007. Botanical Identification of 200 Easter Island Wood Carvings. In proceedings: Migration, Identity, and Cultural Heritage (The Gotland Papers). Gotland University, Sweden. 125-139.

Orliac, C. \& M. Orliac. 1998. Composition et Evolution de la

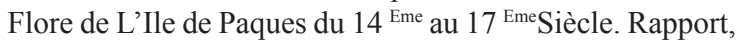
CNRS, UMR 1730.

Orliac C. \& M. Orliac. 2008. Extinct flora of Easter Island. In At the heart of Ancient Societies, French contributions to Pacific Archaeology. Eds. A. Di Piazza, E. Pearthreet Sand C. Les Cahiers de l'Archéologie en Nouvelle-Calédonie 18: $197-208$.

Oyarzún, A. 1921. Toromiros de la isla de Pascua. Revista Chilena de Historia. Natural Año XXV: 133-140.

Palmer, J.L. 1870a. A Visit to Easter Island, or Rapa Nui, in 1868. Proceedings of the Royal Geographical Society of London 14(2): 108-120.

PAlmer, J.L. 1870b. A Visit to Easter Island, or Rapa Nui, in 1868. Journal of the Royal Geographical Society of London, 40: 167-181.

Philippi, R. 1873. La isla de Pascua i sus habitantes. Jeografia $\mathrm{N}^{\circ} 2$. Anales de la Universidad de Chile 43:365-434.

PinART, A. 1877. Voyage to Eastern Island - 1877. Early Visitors to Easter Island 1864-1877. The Reports of Eugene Eyraud, Hippolyte Roussel, Pierre Loti \& Alphonse Pinart. Editors G.Lee, A.M.Altman \& F.Morin. The Eastern Island Foundation. 143 pp.

Rapaport, M. 2006. Eden in Peril: Impact of Humans on Pacific Islands Ecosystems. Island Studies in Journal. (1) Number 1: 109-124.

Rolett, B. \& J. Diamond. 2004. Environmental predictors of pre European deforestation on Pacific Islands. Nature 431: 443-446.

Roussel, H. 1869. Easter Island or Rapa Nui Early Visitors to Easter Island 1864-1877. The Reports of Eugene Eyraud, Hippolyte Roussel, Pierre Loti \& Alphonse Pinart. Editors G. Lee, A.M. Altman \& F. Morin. The Eastern Island Foundation.143 pp.

Rull, V., N. Cañellas-Bolta, A. Sáez, S. Giralt, O. Pla \& O. Margalef. 2010. Paleoecology of Eastern Island: Evidences and uncertainties. Earth-Sciences Reviews 99: 50-60.

Skottsberg, C. 1953. The Natural History of Juan Fernandez and 
Easter Island.Vol. I. Geography, Geology and Origin of Island Life. Almqvist \& Wiksells Boktryckeri. Uppsala. 441pp.

Stevenson, C.M., T.M. Jackson, A. Mieth, H.R. Bork \& T. LADEFOGED. 2006. Prehistoric and early historic agriculture at Maunga Orito, Eastern Island (Rapa Nui), Chile. Antiquity 80: 919-936.
Toro, P. 1893. Isla de Pascua. Memoria del Culto i Colonización presentada al Congreso Nacional en 1892. Tomo III, 179216. Santiago, Chile.

Vargas, P., C. Cristino \& R. Izaurieta. 2006. 1.000 años en Rapa Nui: Arqueología del Asentamiento Humano en Isla de Pascua. Editorial Universitaria, Santiago. Chile. 426 pp.

Recibido: 26.01 .12

Aceptado: 21.08 .13 